\title{
Ferromagnetic Systems with Hopping Interaction
}

\author{
G. Górski* AND J. Mizia \\ Institute of Physics, University of Rzeszów, Rejtana 16A, 35-959 Rzeszów, Poland
}

\begin{abstract}
We analyze the influence of hopping interaction on magnetic ordering. Scattering scheme of the Hubbard III approximation with included inter-site kinetic electron-electron correlation is used. The hopping interaction and inter-site correlation lead to two spin dependent effects: the band width correction and the band-shift correction. The band-shift correction factor causes an exchange splitting between the spin-up and spin-down spectrum, and its role is similar to the exchange interaction in the classic Stoner model. The spin dependent band width correction enhanced strongly by the inter-site kinetic correlation lowers the kinetic energy of electrons by decreasing the majority spin band width for some electron occupations with respect to the minority spin band width. The results show that in the case of the symmetrical density of states there is only ferromagnetic enhancement. For the strongly asymmetrical density of states there is a ferromagnetic transition.
\end{abstract}

PACS numbers: 71.10.Fd, 75.10.Lp

\section{Introduction}

The Hubbard model [1] is extensively used to analyze strong electron correlations in the narrow energy bands. The model is used to describe such phenomena as itinerant ferromagnetism, metal-insulator phase transitions, or high- $\mathrm{T}_{\mathrm{C}}$ superconductivity. The classic form of this model describes the behavior of narrow band electrons in the presence of strong single site electron-electron repulsion $U=(i i|1 / r| i i)$. In the systems with strong correlation the on-site Coulomb interaction $U$ causes a split of the spin band into two sub-bands: lower sub-band centered around the atomic level $T_{0}$, and the upper sub-band centered around the level $T_{0}+U$. The exact solution of the Hubbard model was obtained only in some specific cases, e.g. for the one-dimensional system. For multi-dimensional systems only the approximate solutions exist. The most established and logically justifiable approximate solution in 3-dimensional (3D) is the Hubbard III approximation [2]. Unfortunately, this approximation did not produce the ferromagnetic ground state [3]. The problem whether there is a magnetic ordering in 3D Hubbard model is still unsolved.

In our previous paper [4] we described in great detail the Hubbard III approximation applied to the basic Hubbard model with interaction $U$ only, and with the included inter-site kinetic correlation functions $\left\langle c_{i-\sigma}^{+} c_{j-\sigma}\right\rangle$ and $\left\langle\hat{n}_{i \sigma} c_{i-\sigma}^{+} c_{j-\sigma}\right\rangle$. In effect we have obtained the band width and the band-shift corrections of the original electron band. The band-shift correction causes an exchange splitting between the spin-up and spin-down spectrum, and the spin dependent band width correction lowers the kinetic energy of electrons by decreasing the majority spin band width with respect to the minority spin band width.

* corresponding author; e-mail: ggorski@univ.rzeszow.pl
The realistic systems where ferromagnetism exists were described using the extended Hubbard model where in addition to the basic Coulomb on-site interaction $U$ the different inter-site interactions $V_{i j m n}=(i j|1 / r| m n)$ were added. One of these interactions used in the description of magnetic ordering is the correlated hopping interaction $\Delta t=(i, i|1 / r| j, i)$. The role of hopping interaction in creating the ferromagnetic state was analyzed predominantly in the mean field approach, which obviously led to the ferromagnetism [5-7]. In this paper we present the model in which the inter-site kinetic correlation $\left\langle c_{i-\sigma}^{+} c_{j-\sigma}\right\rangle$ and the hopping interaction $\Delta t=(i, i|1 / r| j, i)$ are included together into the Hubbard III approximation within the scattering effect scheme. We obtain the band-shift and band width corrections to the classic Hubbard III or coherent potential approximation (CPA) solution which are created by the hopping interaction. In the case of strongly asymmetrical density of states (DOS) they can lead to the spontaneous ferromagnetic ordering.

\section{The model}

We analyze the basic Hubbard model with added hopping interaction and the exchange field

$$
\begin{aligned}
H & =-\sum_{i j \sigma} t_{i j} c_{i \sigma}^{+} c_{j \sigma}+\frac{U}{2} \sum_{i \sigma} \hat{n}_{i \sigma} \hat{n}_{i-\sigma} \\
& +\sum_{i j \sigma} \Delta t_{i j}\left(\hat{n}_{i-\sigma}+\hat{n}_{j-\sigma}\right) c_{i \sigma}^{+} c_{j \sigma} \\
& -\sum_{i \sigma}\left(\mu+F_{i n} n_{\sigma}\right) \hat{n}_{i \sigma},
\end{aligned}
$$

where $t_{i j}$ - hopping integral between the $i$-th and $j$-th lattice site, $U$ is the on-site Coulomb interaction, $\Delta t_{i j}$ is the hopping interaction, $F_{i n}$ is the on-site atomic Stoner field (exchange field) in the Hartree-Fock (H-F) approximation and $\mu$ is the chemical potential. The operator $c_{i \sigma}^{+}\left(c_{i \sigma}\right)$ is creating (annihilating) an electron with spin 
$\sigma=\uparrow, \downarrow$ on the $i$-th lattice site, $\hat{n}_{i \sigma}=c_{i \sigma}^{+} c_{i \sigma}$ is the electron number operator for electrons with spin $\sigma$ on the $i$-th lattice site. In the many-body considerations below, the term with chemical potential and with on-site atomic Stoner field will be absent, since they will be moved into the Fermi-Dirac statistics (see Eq. (13)).

To analyze Hamiltonian (1) we use the equation of motion for the Green functions. For the single electron
Green function we can write the expression [2]:

$$
\left\langle\left\langle c_{i \sigma} ; c_{j \sigma}^{+}\right\rangle\right\rangle_{\varepsilon}=\sum_{\alpha= \pm}\left\langle\left\langle\hat{n}_{i-\sigma}^{\alpha} c_{i \sigma} ; c_{j \sigma}^{+}\right\rangle\right\rangle_{\varepsilon} .
$$

After applying Hubbard [2] scattering correction scheme with included non-zero inter-site correlation the equation of motion for the Green functions $\left\langle\left\langle\hat{n}_{i-\sigma}^{\alpha} c_{i \sigma} ; c_{j \sigma}^{+}\right\rangle\right\rangle_{\varepsilon}$ $(\alpha= \pm)$ takes on the following form:

$$
\begin{aligned}
& \left(\varepsilon-\varepsilon_{\alpha}-S_{\Delta t, \sigma}\right)\left\langle\left\langle\hat{n}_{i-\sigma}^{\alpha} c_{i \sigma} ; c_{j \sigma}^{+}\right\rangle\right\rangle_{\varepsilon}=n_{-\sigma}^{\alpha}\left(\delta_{i j}-\sum_{l} t_{i l}\left\langle\left\langle c_{l \sigma} ; c_{j \sigma}^{+}\right\rangle\right\rangle_{\varepsilon}\right)-\sum_{l} t_{i l}\left\langle\left\langle\left(\hat{n}_{i-\sigma}^{\alpha}-n_{-\sigma}^{\alpha}\right) c_{l \sigma} ; c_{j \sigma}^{+}\right\rangle\right\rangle_{\varepsilon} \\
& \quad+\sum_{l} \sum_{\beta= \pm}\left(\Delta t_{i l}^{\alpha}+\Delta t_{i l}^{\beta}\right)\left\langle\left\langle\left(\hat{n}_{i-\sigma}^{\alpha}-n_{-\sigma}^{\alpha}\right) \hat{n}_{l-\sigma}^{\beta} c_{l \sigma} ; c_{j \sigma}^{+}\right\rangle\right\rangle_{\varepsilon}+n_{-\sigma}^{\alpha} \sum_{l} \sum_{\beta= \pm}\left(\Delta t_{i l}^{\alpha}+\Delta t_{i l}^{\beta}\right)\left\langle\left\langle\hat{n}_{l-\sigma}^{\beta} c_{l \sigma} ; c_{j \sigma}^{+}\right\rangle\right\rangle_{\varepsilon}
\end{aligned}
$$

where $S_{\Delta t, \sigma}$ is the inter-site correlation parameter creating the relative shift of spin bands

$$
S_{\Delta t, \sigma}=\frac{1}{N} \sum_{l i} \Delta t_{i l}\left\langle c_{i-\sigma}^{+} c_{l-\sigma}+c_{l-\sigma}^{+} c_{i-\sigma}\right\rangle .
$$

The notation of $\hat{n}_{i \sigma}^{\alpha}, n_{\sigma}^{\alpha}$, and $\varepsilon_{\alpha}$ follows the original Hubbard paper [2], and that for the hopping interaction is the following: $\Delta t_{i l}^{+}=\Delta t_{i l}$ and $\Delta t_{i l}^{-}=0$. To solve Eq. (3) we find the functions $\left\langle\left\langle\hat{n}_{i-\sigma}^{\alpha} c_{l \sigma} ; c_{j \sigma}^{+}\right\rangle\right\rangle_{\varepsilon}$ and $\left\langle\left\langle\left(\hat{n}_{i-\sigma}^{\alpha}-\right.\right.\right.$ $\left.\left.\left.n_{-\sigma}^{\alpha}\right) \hat{n}_{l-\sigma}^{\beta} c_{l \sigma} ; c_{j \sigma}^{+}\right\rangle\right\rangle_{\varepsilon}$ using the scattering effect scheme and we obtain the following set of equations:

$$
\begin{aligned}
& {\left[\begin{array}{cc}
\varepsilon^{\prime}-\varepsilon_{+}^{\prime}+2 n_{-\sigma}^{+} \gamma^{+}\left(\varepsilon_{\boldsymbol{k}}-T_{0}\right) & n_{-\sigma}^{+}\left(\gamma^{+}+\gamma^{-}\right)\left(\varepsilon_{\boldsymbol{k}}-T_{0}\right) \\
n_{-\sigma}^{-}\left(\gamma^{+}+\gamma^{-}\right)\left(\varepsilon_{\boldsymbol{k}}-T_{0}\right) & \varepsilon^{\prime}-\varepsilon_{-}^{\prime}+2 n_{-\sigma}^{-} \gamma^{-}\left(\varepsilon_{\boldsymbol{k}}-T_{0}\right)
\end{array}\right]\left[\begin{array}{c}
\Gamma_{\boldsymbol{k}, \sigma}^{+}(\varepsilon) \\
\Gamma_{\boldsymbol{k}, \sigma}^{-}(\varepsilon)
\end{array}\right]} \\
& \quad=\left[\begin{array}{c}
n_{-\sigma}^{+} \\
n_{-\sigma}^{-}
\end{array}\right]\left[1+\left(\varepsilon_{\boldsymbol{k}}-T_{0}\right) G_{\boldsymbol{k}}^{\sigma}(\varepsilon)-\lambda_{\sigma}(\varepsilon) G_{\boldsymbol{k}}^{\sigma}(\varepsilon)\right]-\left[\begin{array}{c}
n_{-\sigma}^{+} \Omega_{\sigma}^{+}(\varepsilon) \\
n_{-\sigma}^{-} \Omega_{\sigma}^{-}(\varepsilon)
\end{array}\right] G_{\boldsymbol{k}}^{\sigma}(\varepsilon),
\end{aligned}
$$

where $\gamma^{\alpha}$ is the hopping interaction parameter defined as $\gamma^{\alpha}=\Delta t_{i j}^{\alpha} / t_{i j}, \varepsilon^{\prime}=\varepsilon-S_{\Delta t, \sigma}-\lambda_{\sigma}(\varepsilon)$ and $\varepsilon_{\alpha}^{\prime}=\varepsilon_{\alpha}+\Omega_{\sigma}^{\alpha}(\varepsilon)$. Functions $\Gamma_{\boldsymbol{k}, \sigma}^{\alpha}(\varepsilon)$ are the Fourier transforms of $\left\langle\left\langle\hat{n}_{i-\sigma}^{\alpha} c_{i \sigma} ; c_{j \sigma}^{+}\right\rangle\right\rangle_{\varepsilon}$.

Solving Eqs. (5) we obtain the final relation for the Green function $G_{\boldsymbol{k}}^{\sigma}(\varepsilon)$ :

$$
G_{\boldsymbol{k}}^{\sigma}(\varepsilon)=\frac{1}{\varepsilon-\Sigma_{\mathrm{tot}, \boldsymbol{k}}^{\sigma}(\varepsilon)-\left(\varepsilon_{\boldsymbol{k}}-T_{0}\right)},
$$

where the self-energy $\Sigma_{\text {tot, } \boldsymbol{k}}^{\sigma}(\varepsilon)=\Sigma_{0}^{\sigma}(\varepsilon)+\Sigma_{1, \boldsymbol{k}}^{\sigma}(\varepsilon)$ is the sum of $k$-independent term $\Sigma_{0}^{\sigma}(\varepsilon)$ responsible for the band-shift correction

$$
\Sigma_{0}^{\sigma}(\varepsilon)=n_{-\sigma}^{+} \varepsilon_{+}+n_{-\sigma}^{-} \varepsilon_{-}+S_{\Delta t, \sigma}+\frac{n_{-\sigma}^{-} n_{-\sigma}^{+}\left(\varepsilon_{+}-\varepsilon_{-}\right)\left[\varepsilon_{+}-\varepsilon_{-}+\Omega_{\sigma}^{+}(\varepsilon)-\Omega_{\sigma}^{-}(\varepsilon)\right]}{\varepsilon-S_{\Delta t, \sigma}-\lambda_{\sigma}(\varepsilon)-\left[n_{-\sigma}^{+}\left(\varepsilon_{-}+\Omega_{\sigma}^{-}(\varepsilon)\right)+n_{-\sigma}^{-}\left(\varepsilon_{+}+\Omega_{\sigma}^{+}(\varepsilon)\right)\right]},
$$

and $k$-dependent term $\Sigma_{1, k}^{\sigma}(\varepsilon)$ responsible for the band width correction

$$
\Sigma_{1, \boldsymbol{k}}^{\sigma}(\varepsilon) \equiv-2 n_{-\sigma}^{+} \gamma^{+}\left(\varepsilon_{\boldsymbol{k}}-T_{0}\right)-\frac{n_{-\sigma}^{-} n_{-\sigma}^{+}\left[2\left(\varepsilon_{+}-\varepsilon_{-}\right)+\Omega_{\sigma}^{+}(\varepsilon)-\Omega_{\sigma}^{-}(\varepsilon)-\gamma^{+}\left(\varepsilon_{\boldsymbol{k}}-T_{0}\right)\right] \gamma^{+}\left(\varepsilon_{\boldsymbol{k}}-T_{0}\right)}{\varepsilon-S_{\Delta t, \sigma}-\lambda_{\sigma}(\varepsilon)-\left[n_{-\sigma}^{+}\left(\varepsilon_{-}+\Omega_{\sigma}^{-}(\varepsilon)\right)+n_{-\sigma}^{-}\left(\varepsilon_{+}+\Omega_{\sigma}^{+}(\varepsilon)\right)\right]} .
$$

The self-energy $k$-independent term depends mainly on the Coulomb repulsion $U$ and the $k$-dependent term depends mainly on the hopping interaction. Both these terms $\left(\Sigma_{0}^{\sigma}(\varepsilon)\right.$ and $\left.\Sigma_{1, \boldsymbol{k}}^{\sigma}(\varepsilon)\right)$ are complex leading to the finite lifetime of quasi-particles. The self-energy $\Sigma_{1, \boldsymbol{k}}^{\sigma}(\varepsilon)$ causes decrease of the band width and increase in the DOS. The self-energy $\Sigma_{0}^{\sigma}(\varepsilon)$ causes the shift of the spin bands.

The parameters $\lambda_{\sigma}(\varepsilon)$ and $\Omega_{\sigma}^{\alpha}(\varepsilon)$ used above are given as

$$
\lambda_{\sigma}(\varepsilon)=\varepsilon-T_{0}-\Sigma_{0}^{\sigma}(\varepsilon)-\frac{1}{G_{i i}^{\sigma}(\varepsilon)} \frac{1}{N} \sum_{\boldsymbol{k}} \frac{\varepsilon-\Sigma_{0}^{\sigma}(\varepsilon)-\left(\varepsilon_{\boldsymbol{k}}-T_{0}\right)}{\varepsilon-\Sigma_{\text {tot }, \boldsymbol{k}}^{\sigma}(\varepsilon)-\left(\varepsilon_{\boldsymbol{k}}-T_{0}\right)},
$$

and 


$$
\begin{aligned}
& \Omega_{\sigma}^{\alpha}(\varepsilon)=\left[\frac{\gamma^{\alpha}}{\varepsilon-\Sigma_{0}^{\sigma}(\varepsilon)}+\frac{\gamma^{+} n_{-\sigma}^{+}}{\varepsilon-\varepsilon_{+}-S_{\Delta t, \sigma}-n_{-\sigma}^{-}\left(\lambda_{\sigma}(\varepsilon)+\Omega_{\sigma}^{-}(\varepsilon)\right)}\right] \\
& \quad \times\left\{\frac{1}{N} \sum_{\boldsymbol{k}}\left(\varepsilon_{\boldsymbol{k}}-T_{0}\right)\left[\varepsilon_{\boldsymbol{k}}-T_{0}+\Sigma_{1, \boldsymbol{k}}^{\sigma}(\varepsilon)\right]+\frac{1}{G_{i i}^{\sigma}(\varepsilon)}\left[T_{0}-\left(\varepsilon-\Sigma_{0}^{\sigma}(\varepsilon)\right) \frac{1}{N} \sum_{\boldsymbol{k}} \frac{\varepsilon_{\boldsymbol{k}}-T_{0}}{\varepsilon-\Sigma_{\mathrm{tot}, \boldsymbol{k}}^{\sigma}(\varepsilon)-\left(\varepsilon_{\boldsymbol{k}}-T_{0}\right)}\right]\right\},
\end{aligned}
$$

where

$$
G_{l m}^{\sigma}(\varepsilon)=\frac{1}{N} \sum_{\boldsymbol{k}} \frac{\exp \left(\mathrm{i} \boldsymbol{k} \cdot\left(\boldsymbol{R}_{l}-\boldsymbol{R}_{m}\right)\right)}{\varepsilon-\Sigma_{\mathrm{tot}, \boldsymbol{k}}^{\sigma}(\varepsilon)-\left(\varepsilon_{\boldsymbol{k}}-T_{0}\right)} .
$$

\section{Results and conclusions}

In further analysis we will use two coupled equations for electron number and magnetization

$$
n=n_{\uparrow}+n_{\downarrow}, \quad m=n_{\uparrow}-n_{\downarrow},
$$

where $n_{ \pm \sigma}$ are given by

$$
\begin{aligned}
n_{\sigma} & =-\int_{-\infty}^{\infty} \frac{1}{\pi} \operatorname{Im}\left[\frac{1}{N} \sum_{\boldsymbol{k}} G_{\boldsymbol{k}}^{\sigma}(\varepsilon)\right] \\
& \times\left[1+\exp \left(\left(\varepsilon-\mu-F_{i n} n_{\sigma}\right) / k_{\mathrm{B}} T\right)\right]^{-1} \mathrm{~d} \varepsilon .
\end{aligned}
$$

In the numerical calculations we will use the asymmetrical DOS of the type [8]:

$$
\rho(\varepsilon)=\frac{1+\sqrt{1-a^{2}}}{\pi D} \frac{\sqrt{D^{2}-\varepsilon^{2}}}{D+a \varepsilon} .
$$

The calculated values of magnetization depend on $F_{\text {in }}$. For $m \rightarrow 0$ we obtain the critical value of the on-site exchange interaction $F_{i n}^{\mathrm{cr}}$. This value will identify the paramagnetic static susceptibility which is given by [9]:

$$
\chi=2 \mu_{\mathrm{B}} /\left(F_{i n}^{\mathrm{cr}}-F_{\text {in }}\right) .
$$

The constant $F_{i n}$ is set by the material but in this report we put rather rigorous condition for this constant to be zero. As a result the susceptibility will grow when $F_{i n}^{\text {cr }}$ will decrease.

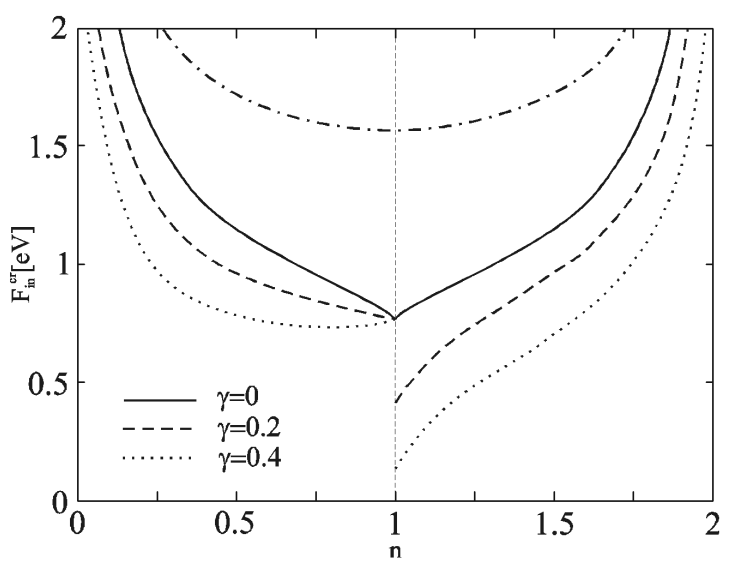

Fig. 1. Critical value of the on-site exchange interaction $F_{i n}^{\mathrm{cr}}$ as function of electron concentration $n$ calculated for the semi-elliptic DOS $(a=0)$ at different values of the hopping parameter $\gamma$. The Coulomb repulsion $U=5 D$, and $D=1 \mathrm{eV}$. The Stoner critical field $F_{i n}^{\mathrm{cr}}=1 / \rho\left(\varepsilon_{\mathrm{F}}\right)$ is added in the case of $\gamma=0$ and $U=0$ (dot-dash line).

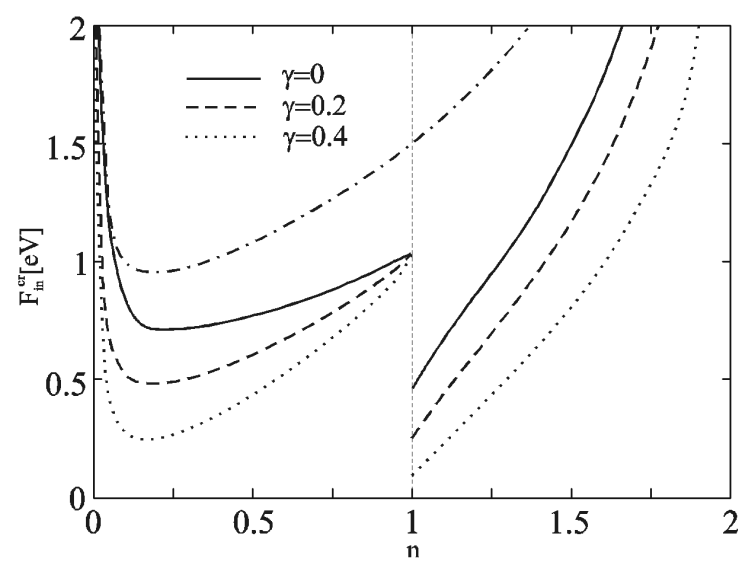

Fig. 2. Critical value of the on-site exchange interaction $F_{i n}^{\mathrm{cr}}$ as function of electron concentration $n$ calculated for the asymmetrical DOS $(a=0.9)$ at different values of the hopping parameter $\gamma$. The Coulomb repulsion $U=5 D$, and $D=1 \mathrm{eV}$. The Stoner critical field $F_{i n}^{\text {cr }}=1 / \rho\left(\varepsilon_{\mathrm{F}}\right)$ is added as the dot-dash line.

In Fig. 1 we present the dependence of $F_{i n}^{\text {cr }}$ on electron concentration for the symmetrical semi-elliptic DOS $(a=0)$. The results show that the exchange field $F_{i n}^{\text {cr }}$ required for ferromagnetism has a strong decrease under the influence of hopping interaction $\Delta t$ as compared to the classic Hubbard III result, where only the on-site $U$ interaction was taken into account. The dependence in the presence of additional to $U$ hopping interaction is not symmetrical since the hopping interaction $\Delta t$ is the off-diagonal Coulomb interaction. For comparison the classic Stoner result is added in the figure.

The decrease of the exchange field comes from: shift of the spin bands by a factor of $S_{\Delta t, \sigma}$, change in the DOS with concentration, change in the band width of spin bands with magnetization. The joint result of these three effects depends on electron concentration. At $n<1$ the decrease of $F_{i n}^{\mathrm{cr}}$ is caused mainly by the band shift. At $n>1$ the decrease of $F_{i n}^{\text {cr }}$ is driven by both: the band shift and the strong change in the width of spin bands.

For the asymmetric bands with $a \neq 0$ we obtain smaller values of $F_{i n}^{\text {cr }}$. They are shown in Fig. 2 for different values of the hopping parameter $\gamma$ and for the asymmetry parameter $a=0.9$. Comparing these results with the results for the semi-elliptic band we see that for electron concentration $n \approx 1^{-}$we get the increase of $F_{i n}^{\mathrm{cr}}$, while for $n \approx 1^{+}$the decrease of $F_{i n}^{\text {cr }}$. The discontinuity of $F_{i n}^{\text {cr }}$ at $n \approx 1$ is caused by different values of DOS on the 
Fermi level at the top of lower sub-band and the bottom of upper sub-band in the case of $a \neq 0$.

The present results for hopping interaction $\Delta t$ included within the Hubbard III scheme show that for the symmetrical DOS there is only the ferromagnetic enhancement of susceptibility (see Eq. (15)). For the asymmetrical DOS under certain conditions (see above) there is a ferromagnetic transition.

\section{References}

[1] J. Hubbard, Proc. R. Soc. A 276, 238 (1963).

[2] J. Hubbard, Proc. R. Soc. A 281, 401 (1964).
[3] J. Mizia, Phys. Status Solidi B 74, 461 (1976).

[4] G. Górski, J. Mizia, Phys. Rev. B 79, 064414 (2009).

[5] J.C. Amadon, J.E. Hirsch, Phys. Rev. B 54, 6364 (1996).

[6] G. Górski, J. Mizia, Physica B 344, 231 (2004).

[7] L. Didukh, Yu. Skorenkyy, V. Hankevych, O. Kramar, Phys. Rev. B 64, 144428 (2001).

[8] J. Wahle, N. Blümer, J. Schlipf, K. Held, D. Vollhardt, Phys. Rev. B 58, 12749 (1998).

[9] J. Mizia, G. Górski, Models of Itinerant Ordering in Crystals: An Introduction, Elsevier Ltd., London 2007, Ch. 7. 